\title{
-NOTES-
}

\section{ON PLANE ELASTIC STRAIN IN DOUBLY-CONNECTED DOMAINS*}

\author{
BY W. PRAGER (Brown University)
}

1. Introduction. The stresses associated with a state of plane elastic strain can be expressed in terms of the second derivatives of Airy's stress function. If $x_{1}, x_{2}, x_{3}$ are rectangular Cartesian coordinates, the axis of $x_{3}$ being normal to the plane of strain, the stress function $\phi\left(x_{1}, x_{2}\right)$ satisfies the differential equation $\Delta^{2} \phi=0$ $\left(\Delta=\partial^{2} / \partial x^{2}+\partial^{2} / \partial y^{2}\right)$, and the given stresses on the boundary determine the tangent planes of the stress surface $x_{3}=\phi\left(x_{1}, x_{2}\right)$ at all points of the boundary, when one such tangent plane is known for each bounding curve. In the case of a singly-connected domain only one such tangent plane must be known, and it can be chosen arbitrarily because the stresses define the stress function only to within an arbitrary linear function of $x_{1}$ and $x_{2}$. In the case of a doubly-connected domain, however, two such tangent planes must be known, and only one of them can be chosen arbitrarily. This paper is concerned with the determination of the second tangent plane in the case where one of the boundary curves is free from loads. Equations from which this tangent plane can be determined, were derived by J. H. Michell ${ }^{1}$ from the condition that the displacements must be single-valued. In the present paper it will be shown that Michell's equations are the natural boundary conditions of the variational problem for the stress function. This remark is of importance when the direct methods of the calculus of variations are used to determine the stress function for a doublyconnected domain. ${ }^{2}$

2. Notations. Basic relations. Throughout this paper Latin subscripts will have the range 1, 2, 3, Greek subscripts the range 1, 2, and the summation convention for repeated subscripts will be used. The rectangular Cartesian coordinates $x_{i}$ are chosen so that the axis of $x_{3}$ is normal to the plane of strain; the position of the origin and the directions of $x_{1}$ and $x_{2}$ are arbitrary. Let $e_{i j}$ be the strain tensor and $s_{i j}$ the reduced stress tensor, i.e. the stress tensor divided by Young's modulus. The stressstrain relations can then be written in the form

$$
e_{i j}=(1+\sigma) s_{i j}-\sigma s_{k k} \delta_{i j}
$$

where $\sigma$ denotes Poisson's ratio, and $\delta_{i j}$ is the Kronecker delta. For the state of plane strain under consideration the condition that $e_{3 s}=0$ gives

$$
s_{33}=\sigma s_{\gamma \gamma} \text {. }
$$

The equations of equilibrium in the plane of strain are

* Received Aug. 7, 1945.

${ }^{2}$ J. H. Michell, Proc. London Math. Soc. (1) 31, 100-146 (1899), Eqs. (13).

2 The necessity of investigating the relations between the natural boundary conditions and Michell's equations arose in connection with work done under a contract in Applied Mechanics for Watertown Arsenal. The author is indebted to the authorities of Watertown Arsenal for the release of this note for publication. 


$$
s_{\gamma \alpha, \gamma}=0,
$$

where the comma followed by the subscript $\gamma$ denotes partial differentiation with respect to $x_{\gamma}$. Equation (3) can be satisfied by setting

$$
s_{\alpha \beta}=\epsilon_{\alpha \lambda \epsilon_{\beta \mu} \phi, \lambda \mu},
$$

where $\phi=\phi\left(x_{1}, x_{2}\right)$ is Airy's stress function, and $\epsilon_{11}=\epsilon_{22}=0, \epsilon_{12}=-\epsilon_{21}=1$. Since $\epsilon_{\alpha \lambda} \epsilon_{\alpha \mu}=\delta_{\lambda \mu}$, the invariant $s_{\alpha \alpha}$ equals $\Delta \phi=\phi_{, \alpha \alpha}$. For a state of plane elastic strain $\Delta s_{\alpha \alpha}=0$, or $\Delta^{2} \phi=0$, i.e. the stress function is biharmonic.

On the boundary of the domain under consideration the surface stresses $f_{\alpha}$ are given. If $n_{\alpha}$ is the unit vector along the outward normal of the boundary, we have $f_{\alpha}=s_{\gamma \alpha} n_{\gamma}=\epsilon_{\gamma \lambda} \epsilon_{\alpha \mu} \phi_{, \lambda \mu} n_{\gamma}$. Now $\epsilon_{\gamma \lambda} n_{\gamma}=t_{\lambda}$, the unit vector of the tangent of the boundary. Accordingly,

$$
f_{\alpha}=\epsilon_{\alpha \mu} \phi, \lambda \mu t_{\lambda}=\epsilon_{\alpha \mu} \partial \phi, \mu / \partial s,
$$

where $\partial / \partial s$ denotes differentiation in the direction of the tangent vector $t_{\lambda}$. Multiplying both sides of (5) by $\epsilon_{\alpha \beta}$ and integrating along the boundary, we obtain

$$
\phi, \beta(s)=\epsilon_{\alpha \beta} \int_{0}^{s} f_{\alpha}(s) d s+\phi_{, \beta}(0) .
$$

The given surface forces $f_{\alpha}$ are thus seen to determine the gradient $\phi_{, \beta}(s)$ of the stress function along a bounding curve, when the gradient $\phi_{, \beta}(0)$ at one point of this curve is known. In other terms, the stress function $\phi$ and its normal derivative $\partial \phi / \partial n$ are defined at all points of a bounding curve, when $\phi$ and its gradient are known at a single point of this curve.

If, in particular, one of the bounding curves is free from loads, the stress function $\phi$ and its normal derivative along this curve equal the values of a linear function $a_{\alpha} x_{\alpha}+b$ and of its normal derivative. Establishing the boundary conditions for the stress function along a bounding curve which is free from loads is therefore equivalent to determining the three coefficients $a_{1}, a_{2}, b$ of this linear function.

3. The variational problem for the stress function. To the strain $e_{i j}$ and the reduced stress $s_{i j}$ corresponds the reduced elastic energy $U=\frac{1}{2} e_{i j} s_{i j}$. In the case of plane strain this energy equals

$$
U=\frac{1}{2}\left[(1+\sigma) s_{i j} s_{i j}-\sigma s_{i i} s_{j j}\right]=\frac{1}{2}(1+\sigma)\left[s_{\alpha \beta} s_{\alpha \beta}-\sigma s_{\alpha \alpha} s_{\beta \beta}\right],
$$

in view of Eqs. (1) and (2). In terms of the stress function introduced in (4) the energy is

$$
U=\frac{1}{2}(1+\sigma)\left[\phi_{, \alpha \beta} \phi_{, \alpha \beta}-\sigma \phi_{, \alpha \alpha} \phi_{, \beta \beta}\right] .
$$

According to the variational principle for the stresses the stress function corresponding to certain boundary conditions is then singled out from amongst all functions which fulfill these boundary conditions and admit continuous derivatives up to the fourth order, by the fact that it minimizes the integral

$$
V=\int\left[\phi_{, \alpha \beta} \phi_{, \alpha \beta}-\sigma \phi_{, \alpha \alpha} \phi_{, \beta \beta}\right] d \omega,
$$

where $d \omega$ denotes the element of area, and the integration is extended over the entire domain. The condition $\delta V=0$ leads to 


$$
\int\left[\phi_{, \alpha \beta} \delta \phi_{, \alpha \beta}-\sigma \phi_{, \alpha \alpha} \delta \phi_{, \beta \beta}\right] d \omega=0
$$

or

$$
\begin{aligned}
& (1-\sigma) \int \phi_{, \alpha \alpha \beta \beta} \delta \phi d \omega-(1-\sigma) \int \phi_{, \alpha \beta \beta} \delta \phi n_{\alpha} d s \\
& \qquad \int \phi_{, \alpha \beta} \delta \phi_{, \alpha} n_{\beta} d s-\sigma \int \phi_{, \beta \beta} \delta \phi_{, \alpha} n_{\alpha} d s=0 .
\end{aligned}
$$

In the case of a doubly-connected domain with loads on one bounding curve only, the stress function $\phi$ and its gradient can be considered as given on the loaded bounding curve. This curve does therefore not furnish any contribution to the line integrals in (11). On the other bounding curve, we have $\phi=a_{\alpha} x_{\alpha}+b$ and $\phi_{, \alpha}=a_{\alpha}$. In addition to the differential equation for the stress function, $\phi_{, \alpha \alpha \beta \beta}=0$ or $\Delta^{2} \phi=0$, Eq. (11) thus gives the following equation which must be fulfilled on the load-free boundary:

$$
\begin{aligned}
\delta a_{\gamma}\left[(1-\sigma) \int \phi_{, \alpha \beta \beta} x_{\gamma} n_{\alpha} d s-\int \phi_{, \gamma \beta} n_{\beta} d s\right. & \left.+\sigma \int \phi_{, \beta \beta} n_{\gamma} d s\right] \\
& +\delta b\left[(1-\sigma) \int \phi_{, \alpha \beta \beta} n_{\alpha} d s\right]=0 .
\end{aligned}
$$

Since $\delta a_{\gamma}$ and $\delta b$ are independent, the expressions in brackets must vanish separately. The second integral in the first bracket can be transformed as follows

$$
\int \phi_{, \gamma \beta} n_{\beta} d s=\int \phi_{, \beta \beta \gamma} d \omega=\int \phi_{, \beta \beta} n_{\gamma} d s
$$

The first bracket can therefore be written as

$$
(1-\sigma)\left[\int \phi_{, \alpha \beta \beta} x_{\gamma} n_{\alpha} d s-\int \phi_{, \beta \beta} n_{\gamma} d s\right] .
$$

With the use of $n_{\gamma}=\epsilon_{\gamma \alpha} t_{\alpha}$, the second integral can be further transformed as follows

$$
\begin{aligned}
\int \phi_{, \beta \beta} n_{\gamma} d s & =\epsilon_{\gamma \alpha} \int \phi_{, \beta \beta} t_{\alpha} d s=\epsilon_{\gamma \alpha} \int \phi_{, \beta \beta} d x_{\alpha} \\
& =-\epsilon_{\gamma \alpha} \int \phi_{, \beta \beta \delta} x_{\alpha} d x_{\delta}=-\epsilon_{\gamma \alpha} \int \phi_{, \beta \beta} x_{\alpha} t_{\delta} d s .
\end{aligned}
$$

Equation (12) is thus equivalent to

$$
\int\left[x_{\gamma} \frac{\partial}{\partial n}(\Delta \phi)+\epsilon_{\gamma \alpha} x_{\alpha} \frac{\partial}{\partial s}(\Delta \phi)\right] d s=0
$$

and

$$
\int \frac{\partial}{\partial n}(\Delta \phi) d s=0 .
$$

The scalar equivalents of (13) are 


$$
\int\left[x_{1} \frac{\partial}{\partial n}(\Delta \phi)+x_{2} \frac{\partial}{\partial s}(\Delta \phi)\right] d s=0
$$

and

$$
\int\left[x_{2} \frac{\partial}{\partial n}(\Delta \phi)-x_{1} \frac{\partial}{\partial s}(\Delta \phi)\right] d s=0 .
$$

Equations (14), (15) and (16) are Michell's conditions which are thus seen to be the natural boundary conditions of the variational problem for the stress function. The manner in which these equations are used in determining $\phi$ is obvious. Let $\phi_{0}, \phi_{1}, \phi_{2}, \phi_{3}$ be the biharmonic functions defined by the following boundary conditions:

1) $\phi_{0}$ and $\partial \phi_{0} / \partial n$ have the prescribed boundary values on the loaded boundary curve $C_{1}$ and vanish on the other boundary curve $C_{2}$;

2) $\phi_{1}=\partial \phi_{1} / \partial n=0$ on $C_{1}$, $\phi_{1}=x_{1}$ and $\partial \phi_{1} / \partial n=n_{1}$ on $C_{2}$

3) $\phi_{2}=\partial \phi_{2} / \partial n=0$ on $C_{1}$, $\phi_{2}=x_{2}$ and $\partial \phi_{2} / \partial n=n_{2}$ on $C_{2}$

4) $\phi_{3}=\partial \phi_{3} / \partial n=0$ on $C_{1}$, $\phi_{3}=1$ and $\partial \phi, / \partial n=0$ on $C_{2}$.

Substituting

$$
\phi=\phi_{0}+a_{1} \phi_{1}+a_{2} \phi_{2}+b \phi_{3}
$$

into Eqs. (14), (15) and (16), we obtain three linear equations from which $a_{1}, a_{2}$ and $b$ can be determined.

\section{THE CAPACITY OF TWIN CABLE-II*}

By J. W. CRAGGS ANd C. J. TRANTER (Military College of Science, Stoke-on-Trent, England)

1. Introduction. In a recent paper $^{1}$ (subsequently referred to as "I") we have given a method for determining the capacity of two circular wires surrounded by concentric touching dielectric sheaths. The present note gives the extension of the method to the case in which the dielectric sheaths are not in contact. The problem considered is the symmetrical one of two infinite parallel circular wires each of radius $R_{1}$ surrounded by concentric sheaths of radius $R_{2}$ and dielectric constant $K_{1}$, the distance between the centers of the wires being $2 L\left(L>R_{2}\right)$. The dielectric constant of the surrounding medium is taken as $K_{2}$.

2. The equations for solution. In line with the treatment in "I" we replace $R_{2}$ by unity, $R_{1} / R_{2}$ by $a$ and $L / R_{2}$ by $s$; we also write $K_{1} / K_{2}=K$. The potentials $V_{1}, V_{2}$ must therefore satisfy $(i)$ the differential equations

$$
\begin{array}{ll}
\nabla^{2} V_{1}=0, & a \leqq r \leqq 1, \\
\nabla^{2} V_{2}=0, & r \geqq 1, \quad x \geqq 0,
\end{array}
$$

and (ii) the boundary conditions

$$
V_{1}=1
$$

* Received June 19, 1945.

${ }^{1} \mathrm{~J}$. W. Craggs and C. J. Tranter, The capacity of twin cable, Quart. Appl. Math. 3, 268-272 (1945). 